Article

\title{
Preparation, Structure, and Properties of Surface Modified Graphene/Epoxy Resin Composites for Potential Application in Conductive Ink
}

\author{
Youliang Cheng ${ }^{1}$, Qingling Zhang ${ }^{2}$, Changqing Fang ${ }^{1,2, *}$, Jing Chen ${ }^{1}$, Jian $\mathrm{Su}^{1}{ }^{1}$, Kaiyan $\mathrm{Xu}^{1}$, \\ Liangliang $\mathrm{Ai}^{1}$ and Donghong $\mathrm{Liu}^{3, *}$ \\ 1 Faculty of Printing, Packaging Engineering and Digital Media Technology, Xi'an University of Technology, \\ Xi'an 710048, China; chengyouliang@xaut.edu.cn (Y.C.); Chenjing05704@163.com (J.C.); \\ sujianwf@163.com (J.S.); sky3220@163.com (K.X.); 15249203037@163.com (L.A.) \\ 2 School of Mechanical and Precision Instrument Engineering, Xi'an University of Technology, Xi'an 710048, \\ China; 18710485611@163.com \\ 3 Fuli Institute of Food Science, Zhejiang University, Hangzhou 310058, China \\ * $\quad$ Correspondence: fangcq@xaut.edu.cn (C.F.); dhliu@zju.edu.cn (D.L.); Tel.: +86-29-82312038 (C.F.); \\ Fax: $+86-29-82312512$ (C.F.)
}

Received: 22 August 2018; Accepted: 27 October 2018; Published: 30 October 2018

\begin{abstract}
The dispersity of graphene (GE) in the matrix has an important influence on the thermal, mechanical, and electrical properties of its derived composites. In this paper, surface modification with a silane coupling agent and a double injection method were used to improve the dispersity of GE in epoxy resin (EP). The thermal, mechanical, and electrical properties of modified graphene/epoxy resin composites (modified GE/EP) were investigated by the thermogravimetric analysis, a four-probe method, and the tensile and bending strength. The results reveal that these properties of the composites can be improved significantly by using the modified GE as the filler. The surface of the modified GE/EP composite was smooth when the curing temperature was $75{ }^{\circ} \mathrm{C}$. The weight loss of the modified GE/EP composite was lower than that of pure EP. The tensile and bending strength of modified GE/EP-0.07 (0.07 wt \% modified GE) reached 74.65 and 106.21 MPa, respectively. In addition, the resistivity of modified GE/EP-0.1 $(0.1 \mathrm{wt} \%$ modified GE) decreased to $52 \Omega \cdot \mathrm{cm}$, which was lower than that of CB/EP-1 (1 wt \% carbon black, $95 \Omega \cdot \mathrm{cm})$ and Ag/EP-50 (50 wt \% Ag particles, $102 \Omega \cdot \mathrm{cm}$ ). It is worth noting that the percolation threshold of the modified GE/EP composites was $0.025 \mathrm{vol} \%$ modified GE. These results show that the modified GE/EP composites have a potential application in conductive ink when the modified GE is used as the conductive filler.
\end{abstract}

Keywords: surface modified graphene; thermal properties; mechanical properties; conductivity

\section{Introduction}

In recent years, carbon black (CB), carbon nanotubes (CNTs), and graphene (GE) used as conductive fillers have attracted the attention of many researchers [1-3]. Due to the high Young's modulus, excellent thermal property, superior conductivity, high surface area, high strength, and fast mobility of charge carries [4-8], the GE is regarded as the ideal reinforcing material for the composites. Moreover, GE/polymer composites exhibit superior performances in many aspects [9-12], and have been applied in sensors, super-capacitors, magnetic shielding, and memory devices [13]. The GE has an important influence on the properties of the composites according to previous reports. Guo and co-workers found that the Young's modulus and shear modulus of composites increased linearly with the increase of GE content [14]. Chen and co-workers reported that the functionalized GE significantly improved the mechanical, thermal, and tribological properties of 
as-prepared composites [15]. In addition, Alam and co-workers greatly enhanced the mechanical properties of composites by adding 1 vol \% of GE [16]. Moreover, Govorov and co-workers have reported that the conductivity of the GE/EP composites was correlated to their tensile stress after the loading of GE [17].

It is important to develop the preparation method of the GE and GE/polymer composites for exploring their applications. There are several methods that can be employed to synthesize GE, including the reduction of graphene oxide (GO) [18], mechanical exfoliation of graphite [19], chemical vapor deposition [20], and epitaxial growth [21]. The reduction method is the most convenient compared to the others. To prepare GE/polymer composites, melt-blending [22], solution blending [23], and in-situ polymerization [24] are mostly employed. The dispersity of GE in the polymer matrix has an important influence on the properties of the composites, which could be improved by modifying the GE with compatible chemical groups for the polymer matrix. Moreover, the surface modification of GE can also improve the dispersity of GE in solvents $[25,26]$. Therefore, the covalent and non-covalent methods have been developed to modify GE. The non-covalent method can greatly preserve the original structure of GE, but the interactions between the GE surface and the modifiers are weak. Therefore, the covalent method is much more desirable in some application fields [19]. In addition, it is interesting that the GE can be used as the conductive filler and epoxy resin as the binder for the conductive ink. Lee and co-workers reported that conductive ink containing the dispersed GE nanosheets showed a high stability [27]. Wei and co-workers reported that the modified GE of various functional groups could improve the surface tension and adhesion of GE ink [26]. Shulga and co-workers prepared an aqueous suspension of the composite using GO and humic acid, which was used as the ink to print the films with various thicknesses [28]. Nemala and co-workers prepared a lower-cost conductive ink with few-layers GE, as a substitute for a Pt-based counter electrode, decreasing the cost significantly [29]. These studies inspire the GE/EP composites to be further explored in conductive inks.

In this paper, we demonstrate an effective strategy to prepare surface modified GE, which can be used as an excellent filler for conductive ink. The GO was first modified by grafting with a silane coupling agent (KH-560) via the covalent method and then reduced into the GE form. The modified GE can be dispersed homogeneously in organic solvents. Subsequently, the modified GE/EP composites were prepared using a double injection method similar to the previous report [30]. Thus, the dispersity of GE in epoxy resin can be improved significantly by associating the above two methods, and the GE content for preparing GE/EP composites with excellent properties can be reduced. The morphologies, structure, conductivity, thermal, and mechanical properties of as-prepared composites were investigated by different characterization methods. Interestingly, when $0.07 \mathrm{wt} \%$ modified GE was added, the tensile and bending strength of the modified GE/EP composite reached 74.65 and 106.21 MPa, respectively. When $0.1 \mathrm{wt} \%$ modified GE was added, the resistivity of the modified GE/EP composite decreased to $52 \Omega \cdot \mathrm{cm}$.

\section{Experimental}

\subsection{Materials}

Natural graphite flakes (100 meshes) used in this paper were purchased from Qingdao Sunshine Co. Ltd. (Qingdao, Shandong, China). Hydrazine hydrate was purchased from Jinan Aoxiang Chemical Reagent Co. Ltd. (Jinan, China). Acetic acid (36 wt \%) and ethanol were purchased from Sinopharm Chemical Reagent Co. Ltd. (Shanghai, China). KH-560 was purchased from Jinan Huanzheng Co. Ltd. (Jinan, China). Epoxy resin (E-44) was purchased from Xi'an Resin Factory (Xi'an, China). Ag particles were purchased from Guangdong Lingguang New Materials Co. Ltd. (Zhaoqing, China), and their average size was about $1.2 \mu \mathrm{m}$. Carbon black (1500 meshes) was purchased from Dongguan Tangxia Xiangyang Graphite Products Processing Plant (Dongguan, China). 


\subsection{Preparation of Modified GO}

Graphene oxide (GO) derived from natural graphite was prepared by using the modified Hummers' method according to previous reports [31,32]. The preparation of modified GO can be briefly described as follows. The GO $(100 \mathrm{mg})$ powder was dispersed in $100 \mathrm{~mL}$ of ethanol by an ultrasonic dispersion with stirring for $3 \mathrm{~h}$ to form the dispersion. A mixture of KH-560 (20 mg) in $10 \mathrm{~mL}$ deionized water and $36 \mathrm{wt} \%$ acetic acid in $7 \mathrm{~mL}$ water was added to the GO dispersion for $30 \mathrm{~min}$ ultrasonic treatment. Then, the mixture was heat-treated with reflux condensing at $80^{\circ} \mathrm{C}$ for $4 \mathrm{~h}$. The products were collected by centrifuging at 10,000 rpm and further washed wish deionized water three times. Finally, the modified GO was dried in a vacuum oven at $80^{\circ} \mathrm{C}$ for $24 \mathrm{~h}$.

\subsection{Reduction of Modified GO}

The modified GO (50 mg) was dispersed in $100 \mathrm{~mL}$ of deionized water by an ultrasonic dispersion with stirring for $2 \mathrm{~h}$. Then, $50 \mathrm{~mL}$ of pre-prepared $85 \%$ hydrazine hydrate was added to the modified GO dispersion. The mixture was heat-treated with reflux condensing at $95{ }^{\circ} \mathrm{C}$ for $24 \mathrm{~h}$. Then, the products were separated by centrifuging at 10,000 rpm and washed with ethanol three times. Finally, the modified GE was obtained after drying in a vacuum oven at $80^{\circ} \mathrm{C}$ for $24 \mathrm{~h}$.

\subsection{Preparation of Modified GE/EP Composites}

The EP $(5 \mathrm{~g})$ was diluted in the mixed solution of $10 \mathrm{~mL}$ ethanol and $10 \mathrm{~mL}$ butanone. A certain amount of the modified GE was dispersed in $20 \mathrm{~mL}$ ethanol. Then, the above two mixtures were slowly injected into a beaker within $10 \mathrm{~min}$ by using the double injection method with magnetic stirring at $500 \mathrm{rpm}$. The tributyl phosphate $(30 \mathrm{mg}$ ) and polyethylene glycol $(50 \mathrm{mg})$ were added to the mixture containing the modified GE and EP with ultrasonic agitation for $2 \mathrm{~h}$. Subsequently, ethylenediamine $(0.6 \mathrm{~g})$ and dibutyl phthalate $(1.5 \mathrm{~g})$ were added to the mixture. Eventually, the above mixture was poured into a polytetrafluoroethylene (PTFE) mold for curing for $4 \mathrm{~h}$ at different temperatures $(70,75$, and $80^{\circ} \mathrm{C}$ ) and the modified GE/EP composites were obtained. The content of modified GE was set as $0.01,0.03,0.05,0.07$, and $0.1 \mathrm{wt} \%$, respectively. In this paper, CB and Ag particles were also used as the fillers for the contrast, and the as-prepared composites were denoted as $\mathrm{CB} / \mathrm{EP}$ and $\mathrm{Ag} / \mathrm{EP}$. The content of $\mathrm{CB}$ was $0.2,0.4,0.6,0.8$, and $1.0 \mathrm{wt} \%$, respectively. In addition, the content of Ag particles was set as 10, 20,30,40, and $50 \mathrm{wt} \%$, respectively. The as-prepared composite samples were denoted as modified GE/EP- $x(x=0.01,0.03,0.05,0.07$, and 0.1 , respectively), CB/EP- $y(y=0.2,0.4,0.6,0.8$, and 1.0 , respectively), and $\mathrm{Ag} / \mathrm{EP}-z(z=10,20,30,40$, and 50, respectively).

\subsection{Characterization}

Fourier transform infrared spectra (FTIR) of the samples were performed on a NICOLET-10 infrared spectrometer (Thermo Fisher Scientific, Waltham, MA, USA) using $\mathrm{KBr}$ as the sample matrix in the range of $1000-3500 \mathrm{~cm}^{-1}$. The morphologies of the samples were investigated by a scanning electron microscope (SEM Hitachi SU8010, Tokyo, Japan). Raman spectra were obtained on a Raman spectrometer (Horiba JOBIN YVON HR800, Kyoto, Japan) and a $633 \mathrm{~nm}$ argon-ion laser was used for the excitation under ambient conditions. The conductivity of the composites was measured on a four-probe instrument (ST-2258C, Suzhou Jingge Electronic Co., Ltd., Suzhou, China), and the tested samples were cut into dimensions of $20 \mathrm{~mm} \times 10 \mathrm{~mm} \times 3 \mathrm{~mm}$. The resistivity was calculated according to Equation (1):

$$
\rho=R L / A
$$

where $L$ is the distance between the electrodes, $A$ is the cross section area, and $R$ is the measured resistance. The thermal stability of the samples was studied by thermogravimetric analysis (TGA) on a thermogravimetric apparatus (NETZSCH TG209, Selb, Germany) in an $\mathrm{N}_{2}$ atmosphere at the heating rate of $10^{\circ} \mathrm{C} \cdot \mathrm{min}^{-1}$. The tensile and bending strength of the modified GE/EP, CB/EP, and $\mathrm{Ag} / \mathrm{EP}$ composites were tested on a universal testing machine (SHIMADZU AGS-J, Kyoto, 
Japan) according to the standard test methods (ASTM D 638-08 Type I [33] and ASTM D 790-03 [34]). For the tensile strength test, the composite samples were cut into a dumbbell-like shape and the dimension was determined according to type I. In addition, the dimension of the composite samples was $127 \mathrm{~mm} \times 12.7 \mathrm{~mm} \times 3.2 \mathrm{~mm}$ for the bending strength test, the crosshead speed was $2 \mathrm{~mm} \cdot \mathrm{min}^{-1}$, and the loading was $5 \mathrm{kN}$. During the mechanical tests and the conductivity measurements, five samples for each composite were tested to get the average value. Error bars often represent one standard deviation of uncertainty. Since the average values were presented in the graph, the standard error is the appropriate measurement to calculate the error bars. The standard deviation and the standard error were calculated according to the Equations (2) and (3), respectively:

$$
\begin{gathered}
\sigma=\sqrt{\frac{1}{n} \sum_{x=1}^{n}\left(x_{i}-\mu\right)^{2}} \\
\sigma_{\mathrm{s}}=\frac{\sigma}{\sqrt{n}}
\end{gathered}
$$

where $\sigma$ is the standard deviation, $n$ is the number of samples (five in this paper), $x_{i}$ presents the measured sample values, $\mu$ presents the average values, and $\sigma_{\mathrm{s}}$ presents the standard error.

\section{Results and Discussions}

Fourier transform infrared spectra of as-prepared GO, modified GO, and modified GE are shown in Figure 1. The intensity of the adsorption peak at $1087 \mathrm{~cm}^{-1}$, ascribed to the stretching vibration of C-O in modified GO, is enhanced compared with GO. In addition, the adsorption peaks at $992 \mathrm{~cm}^{-1}$ and $1270 \mathrm{~cm}^{-1}$, ascribed to the stretching vibration of $\mathrm{Si}-\mathrm{O}$ and the bending vibration of $\mathrm{Si}-\mathrm{C}$ in the spectra of modified GO, respectively, indicate that the surface of GO is modified successfully by $\mathrm{KH}-560$. The adsorption peak at $1735 \mathrm{~cm}^{-1}$ in the spectra of GO and modified GO was attributed to the stretching vibration of $\mathrm{C}=\mathrm{O}$. The intensity of the adsorption peak for $\mathrm{C}=\mathrm{O}$ in the spectra of $\mathrm{GE}$ is lower than that of modified GO. Moreover, the intensity of the peaks at around 3426 and $3201 \mathrm{~cm}^{-1}$, attributed to the stretching vibration of $-\mathrm{O}-\mathrm{H}$, is weakened significantly. This may be due to the reduction of $\mathrm{GO}$ and the coupling between $-\mathrm{O}-\mathrm{H}$ on the $\mathrm{GO}$ and $-\mathrm{O}-\mathrm{CH}_{3}$ in $\mathrm{KH}-560$.

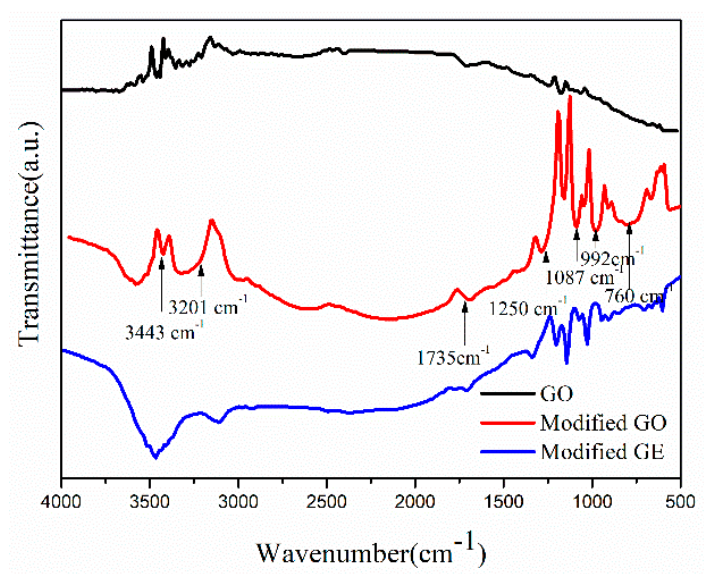

Figure 1. FTIR spectra of GO, modified GO, and modified GE.

The D, G, and G' bands in the Raman spectrum of graphite correspond to the defects of the carbon layers, the graphitic layers, and the overtone of the $\mathrm{D}$ band, respectively. In addition, the intensity ratio of the $\mathrm{D}$ band to $\mathrm{G}$ band $\left(I_{\mathrm{D}} / I_{\mathrm{G}}\right)$ (denoted as $r$ ) reflects the regularity of the crystalline structures in carbon materials $[35,36]$. As shown in Figure 2, the D band at around $1360 \mathrm{~cm}^{-1}$ and $\mathrm{G}$ band at around $1600 \mathrm{~cm}^{-1}$ can be observed in Raman spectra of GO, modified GO, and modified GE, corresponding to the characteristic bands of GE $[37,38]$. Moreover, the calculated $r$ of GO, modified GO, and modified 
GE is $1.08,1.26$, and 1.34, respectively. The $r$ value of modified GE is higher than that of modified GO, suggesting that new graphitic domains have been formed during the reduction of GO [39,40].

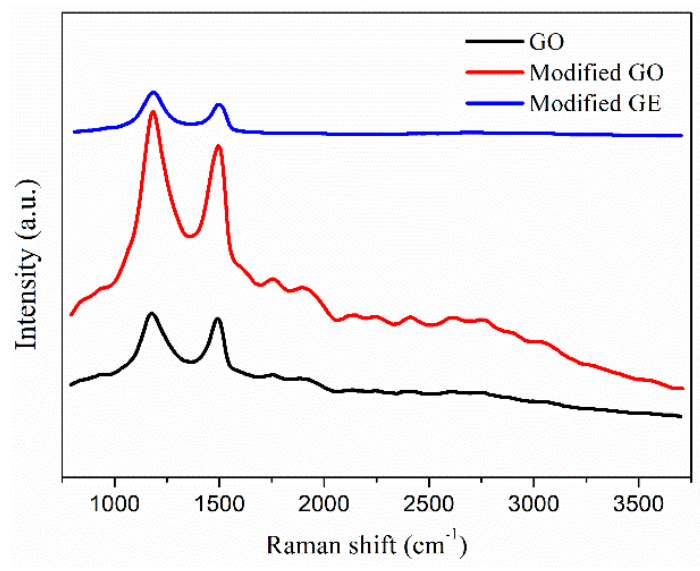

Figure 2. Raman spectra of GO, modified GO, and modified GE.

The as-prepared GO, modified GO, and modified GE dispersions in ethanol after standing for $2 \mathrm{~h}$ are shown in Figure 3. Precipitation was observed for the GO dispersion. However, no precipitation was seen for modified GO and modified GE dispersions, indicating that the surface modification of GO with KH-560 can improve the dispersity of GO in ethanol solution.

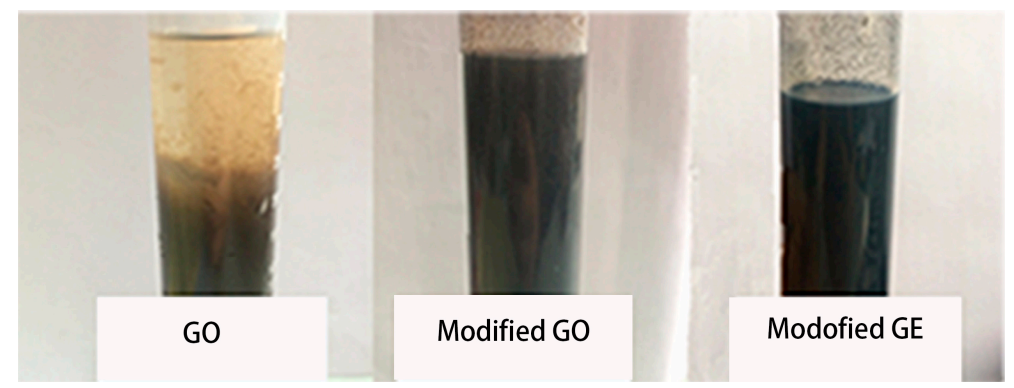

Figure 3. Dispersibility of GO, modified GO, and modified GE in ethanol after standing for $2 \mathrm{~h}$.

For investigating their thermal stability, the thermal degradation of as-prepared samples is evaluated in the range of $50-800{ }^{\circ} \mathrm{C}$. The thermogravimetric and deriveative thermogravimetric curves of GO, modified GO, and modified GE are shown in Figure 4. Although the plots exhibit a similarity in shape, differences are observed in the rate of weight loss. The weight loss of GO has two stages: (1) the removal of water below $180{ }^{\circ} \mathrm{C}$, and (2) the decomposition of the oxygen-containing groups below $800{ }^{\circ} \mathrm{C}$. The total weight loss is $50.89 \mathrm{wt} \%$ in the range of $50-800{ }^{\circ} \mathrm{C}$. The weight loss of modified GO below $190^{\circ} \mathrm{C}$ is ascribed to the removal of water and solvent absorbed on the surface of modified GO. Then, the water and solvent molecules existing between modified GO sheets begin to be removed in the range of $190-270{ }^{\circ} \mathrm{C}$. When the temperature is beyond $270{ }^{\circ} \mathrm{C}$, epoxy groups and other oxygen-containing groups on the modified GO begin to decompose. The total weight loss of modified GO reaches $63.71 \mathrm{wt} \%$ in the range of $50-800{ }^{\circ} \mathrm{C}$. The modified GE exhibits a lower weight loss rate (only $41.29 \mathrm{wt} \%$ ) compared with GO and modified GO. This result demonstrates that there are more functional groups existing on the modified GO and they are decomposed during the heat-treatment. Therefore, the thermal stability of modified GE is the highest and that of modified GO is the lowest. In addition, a higher weight loss rate for modified GO also confirms that KH-560 has been successfully grafted on the surface of GO. 

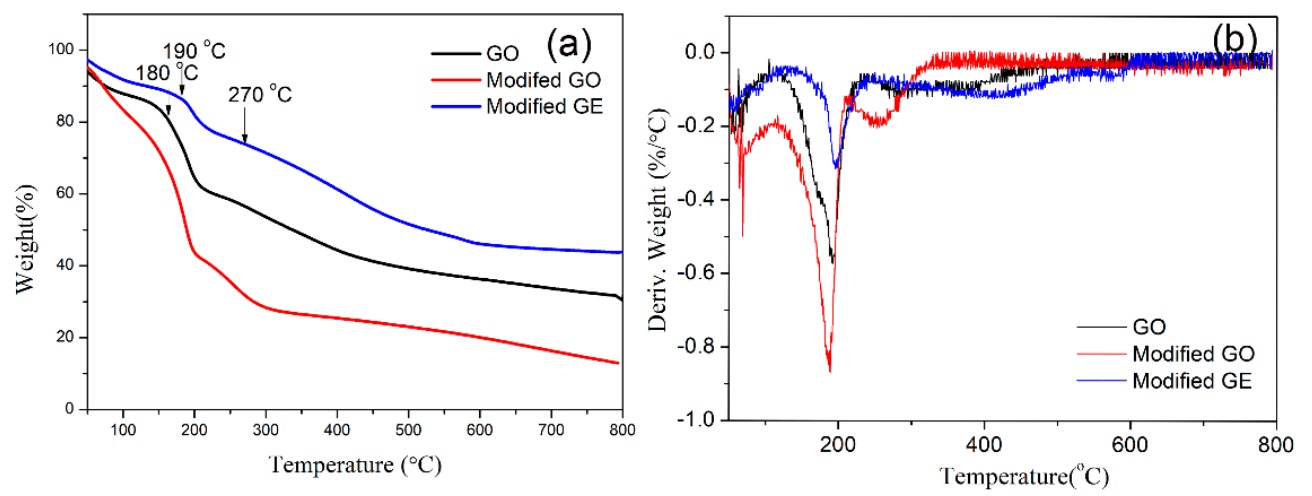

Figure 4. (a) TG of GO, modified GO, and modified GE; (b) DTG of GO, modified GO, and modified GE.

The morphologies of modified GE/EP composites filled with $0.05 \mathrm{wt} \%$ modified GE at different curing temperatures are shown in Figure 5. Lots of bubbles are observed on the surface of modified the GE/EP composite cured at $80^{\circ} \mathrm{C}$. This is due to the quick volatilization of the solvent and the curing agent at a higher temperature compared with other curing temperatures $\left(70\right.$ and $\left.75^{\circ} \mathrm{C}\right)$. The bubbles on the surface of the modified GE/EP composite cured at $70^{\circ} \mathrm{C}$ are obviously reduced (as shown in Figure 5b). However, the upper surface of the composite appears to be transparent. The reason for this may be that the curing velocity of EP is slow at a low curing temperature and the modified GE will settle in the bottom of the EP matrix with a low viscosity. The modified GE will agglomerate, and the phase separation between the fillers and EP matrix has appeared. Thus, the modified GE is distributed unevenly in the EP matrix and the upper surface of the modified GE/EP composites is transparent. As shown in Figure $5 \mathrm{c}$, the surface of the modified GE/EP composite cured at $75{ }^{\circ} \mathrm{C}$ is smooth, indicating that the fillers of modified GE are distributed uniformly in the EP matrix and the appropriate curing temperature of $75^{\circ} \mathrm{C}$. In addition, the illustration diagram for preparing the modified GE/EP composite is shown in Figure 6.

Thermogravimetric analysis is also employed to investigate the thermal stability of the modified GE/EP composite. The weight loss curves of pure EP and modified GE/EP-0.05 in the range of $50-600{ }^{\circ} \mathrm{C}$ at a heating rate of $10^{\circ} \mathrm{C} \cdot \mathrm{min}^{-1}$ are shown in Figure 7 . The total weight loss of pure EP in the range of $50-600{ }^{\circ} \mathrm{C}$ is about $82 \mathrm{wt} \%$ and that of modified GE/EP-0.05 is only about $26 \mathrm{wt} \%$. Compared with pure EP, the thermal stability of the as-prepared composite with modified GE can be improved when modified GE is added to the EP matrix. This is due to the fact that the modified GE with a high specific surface area hinders the removal of low molecular weight fragments in the as-prepared modified GE/EP composite during the thermal decomposition [9,41].
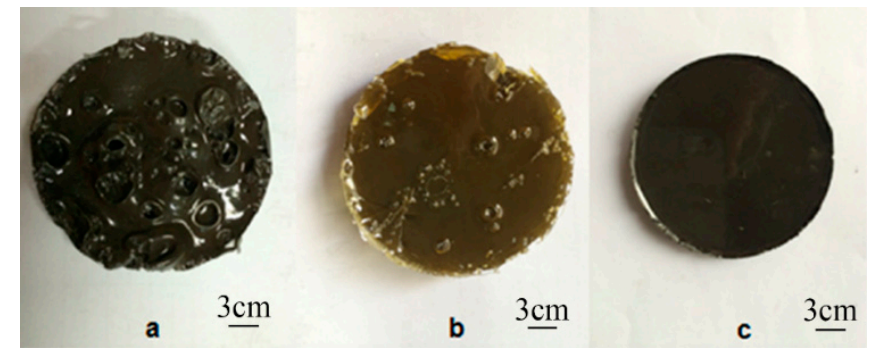

Figure 5. Morphologies of the samples obtained at different curing temperatures: $(\mathbf{a}) 80^{\circ} \mathrm{C},(\mathbf{b}) 70^{\circ} \mathrm{C}$, and (c) $75^{\circ} \mathrm{C}$. 


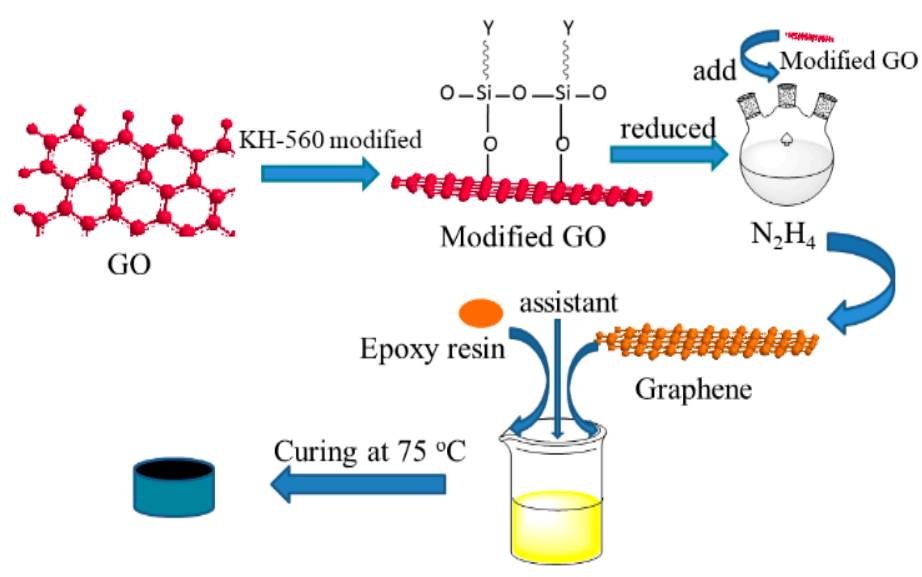

Figure 6. Illustration diagram of the preparation process of the modified GE/EP composite via a double injection method.

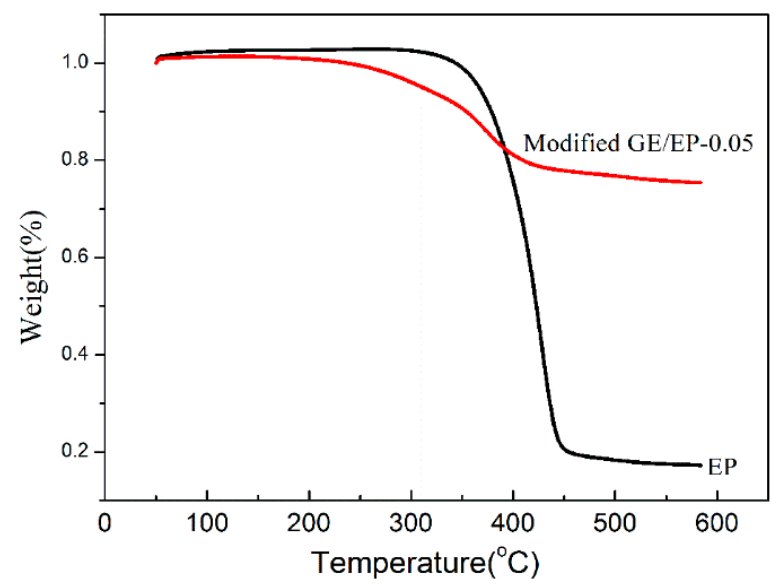

Figure 7. TG of EP and modified GE/EP composite filled with $0.05 \mathrm{wt} \%$ modified GE.

The influences of the fillers content on the mechanical properties of as-prepared modified GE/EP, $\mathrm{CB} / \mathrm{EP}$, and $\mathrm{Ag} / \mathrm{EP}$ composites are shown in Figure 8. When the modified GE content increases from 0.01 to $0.07 \mathrm{wt} \%$, the tensile strength of the as-prepared modified GE/EP composite increases from 58.31 to $74.65 \mathrm{MPa}$ (as shown in Figure 8a). However, the tensile strength begins to decrease when the modified GE content exceeds $0.07 \mathrm{wt} \%$. It can be inferred that the modified GE can improve the tensile strength of the EP matrix. Nevertheless, oxygen-containing groups remaining on the modified GE will react with the curing agent, which decreases the interfacial bonding between modified GE and EP [42]. Tang and co-workers reported that the tensile strength of GE/EP composites was $58 \mathrm{MPa}$ when the GE content was $0.025 \mathrm{wt} \%$ [43]. Chhetri and co-workers found that the tensile strength of the functionalized RGO/EP composites with $0.2 \mathrm{wt} \% \mathrm{RGO}$ could reach $59 \mathrm{MPa}$ [44]. The tensile strength $(58.31 \mathrm{MPa})$ of the as-prepared modified GE/EP composites in this paper is comparable to that in previous reports, but a lower content of filler $(0.01 \mathrm{wt} \%)$ is required. The tensile strength of the $\mathrm{CB} / \mathrm{EP}$ composite increases when increasing the $\mathrm{CB}$ content and it reaches $60.83 \mathrm{MPa}$ when the $\mathrm{CB}$ content is $1.0 \mathrm{wt} \%$ (as shown in Figure $8 \mathrm{~b}$ ). In addition, the tensile strength of the Ag/EP composite increases from 14.18 to $22.48 \mathrm{MPa}$ when the $\mathrm{Ag}$ particles content increases from $10 \mathrm{wt} \%$ to $40 \mathrm{wt} \%$, and then decreases to $18.72 \mathrm{MPa}$ when the Ag particles content is $50 \mathrm{wt} \%$ (as shown in Figure 8c).

As shown in Figure 8d, the bending strength of the composites exhibits a similarity with their tensile strength. The bending strength of the modified GE/EP composite increases from 93.05 to 106.21 MPa when the modified GE content increases from $0.01 \mathrm{wt} \%$ to $0.07 \mathrm{wt} \%$. Because the surface of the modified GE with containing-oxygen groups and $\mathrm{KH}-560$ is wetted fully by EP molecules, the 
filler of modified GE and the matrix of EP can achieve close contact due to the wettability between them. However, the bending strength of the modified GE/EP composite decreases significantly when the modified GE content exceeds $0.07 \mathrm{wt} \%$. As shown in Figure 8e, the bending strength of the $\mathrm{CB} / \mathrm{EP}$ composite increases from 51.27 to $65.49 \mathrm{MPa}$ when increasing the $\mathrm{CB}$ content. The bending strength of the $\mathrm{Ag} / \mathrm{EP}$ composite increases from 21.43 to $34.7 \mathrm{MPa}$ when the Ag particles content increases from $10 \mathrm{wt} \%$ to $40 \mathrm{wt} \%$ and then decreases to $28.03 \mathrm{MPa}$ when it is $50 \mathrm{wt} \%$ (as shown in Figure 8f). When comparing the modified GE/EP composite with the CB/EP and Ag/EP composites, it can be concluded that a small amount of modified GE enables a significant improvement of the mechanical properties for the as-prepared composites. This is ascribed to the interfacial force between the filler of modified GE and the matrix of EP besides the homogeneous dispersion of the filler in the matrix [14]. Thus, the fracture surface of the modified GE/EP composites is investigated to further interpret this phenomenon.
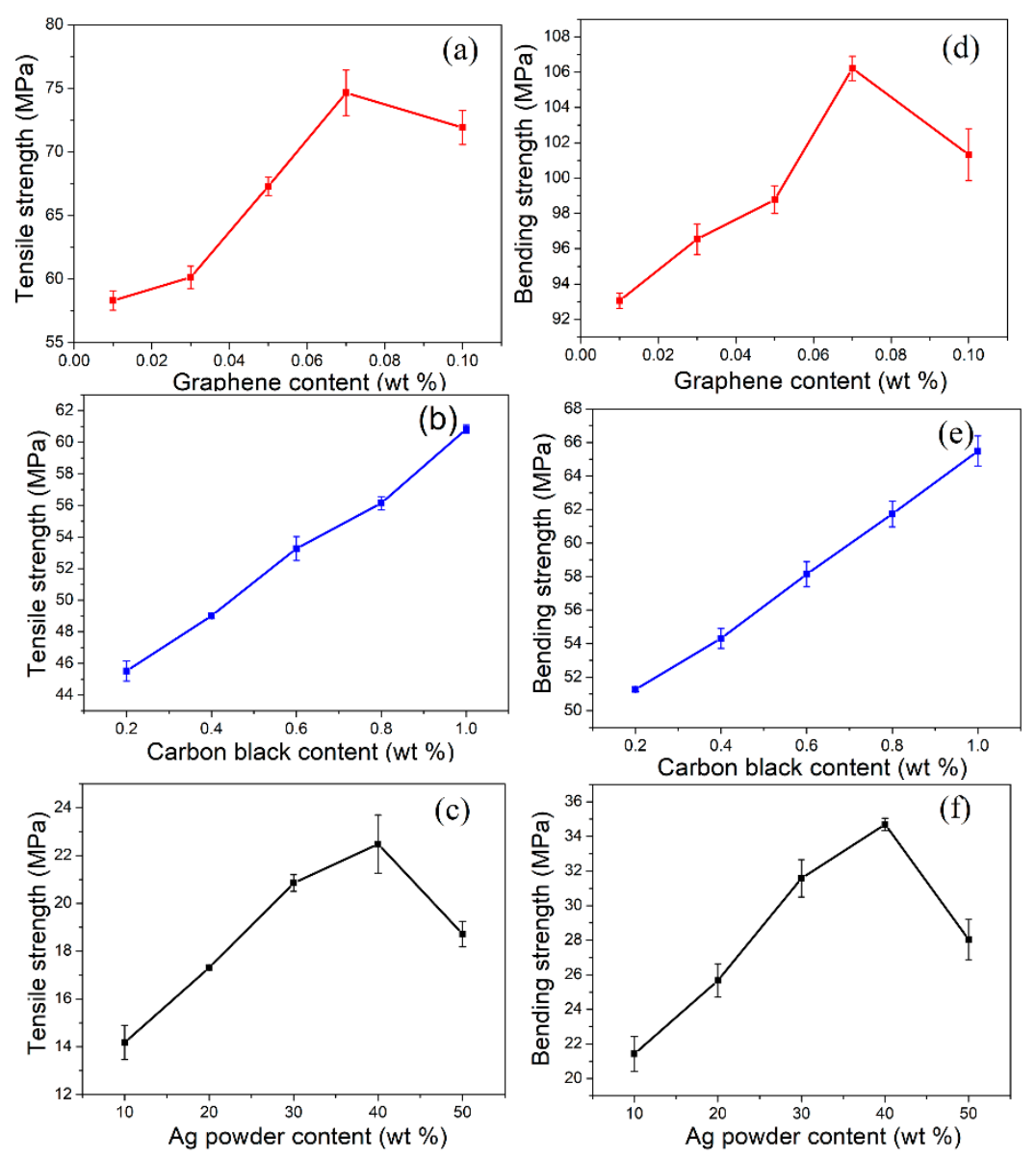

Figure 8. Mechanical properties of the composites: (a) the tensile strength of modified GE/EP composites with different contents of modified GE, (b) the tensile strength of $\mathrm{CB} / \mathrm{EP}$ composites with different contents of $\mathrm{CB},(\mathrm{c})$ the tensile strength of $\mathrm{Ag} / \mathrm{EP}$ composites with different contents of Ag particles, (d) the bending strength of modified GE/EP composites with different contents of modified GE, (e) the bending strength of $\mathrm{CB} / \mathrm{EP}$ composites with different contents of $\mathrm{CB}$, (f) the bending strength of $\mathrm{Ag} / \mathrm{EP}$ composites with different contents of Ag particles.

The fracture surfaces of the modified GE/EP composites with different contents of modified GE are shown in Figure 9. When the modified GE content is $0.03 \mathrm{wt} \%$, there are no obvious sheets on the fracture surface of modified GE/EP-0.03, except for the circle regions (as shown in Figure 9a). When the modified GE content is $0.05 \mathrm{wt} \%$ and $0.07 \mathrm{wt} \%$, many small sheets appear on the fracture surface of modified GE/EP-0.05 and modified GE/EP-0.07, where some cured EP is stripped from the fracture 
surface (as shown in Figure 9b,c). Compared with modified GE/EP-0.03, modified GE/EP-0.05, and modified GE/EP-0.07, the sheet surfaces of modified GE/EP-0.1 are smoother (as shown in Figure 9d). The fracture surface of the modified GE/EP composites also indicates that modified GE has been dispersed homogeneously in the EP matrix, agreeing with the results of the mechanical properties. Compared with $\mathrm{CB} / \mathrm{EP}-0.4$ and $\mathrm{Ag} / \mathrm{EP}-20$, the fracture surfaces of modified GE/EP composites are smoother, demonstrating that the compatibility between the modified GE and EP is better than that for the $\mathrm{CB} / \mathrm{EP}$ and $\mathrm{Ag} / \mathrm{EP}$ composites. Based on the mechanical properties and the fracture surface, it can be concluded that the modified GE is dispersed evenly in the EP matrix when the content is in the range of $0.03 \mathrm{wt} \%-0.07 \mathrm{wt} \%$. Moreover, the appropriate content of the modified GE can improve the mechanical properties of as-prepared composites due to the favorable dispersity of the modified GE in the EP matrix and the strong interfacial force between the modified GE and EP. When the modified GE content is excessive, it is not conducive to improving the mechanical properties due to the inhomogeneous dispersion of the fillers in the matrix, and the fracture surface without stripped EP becomes smoother.
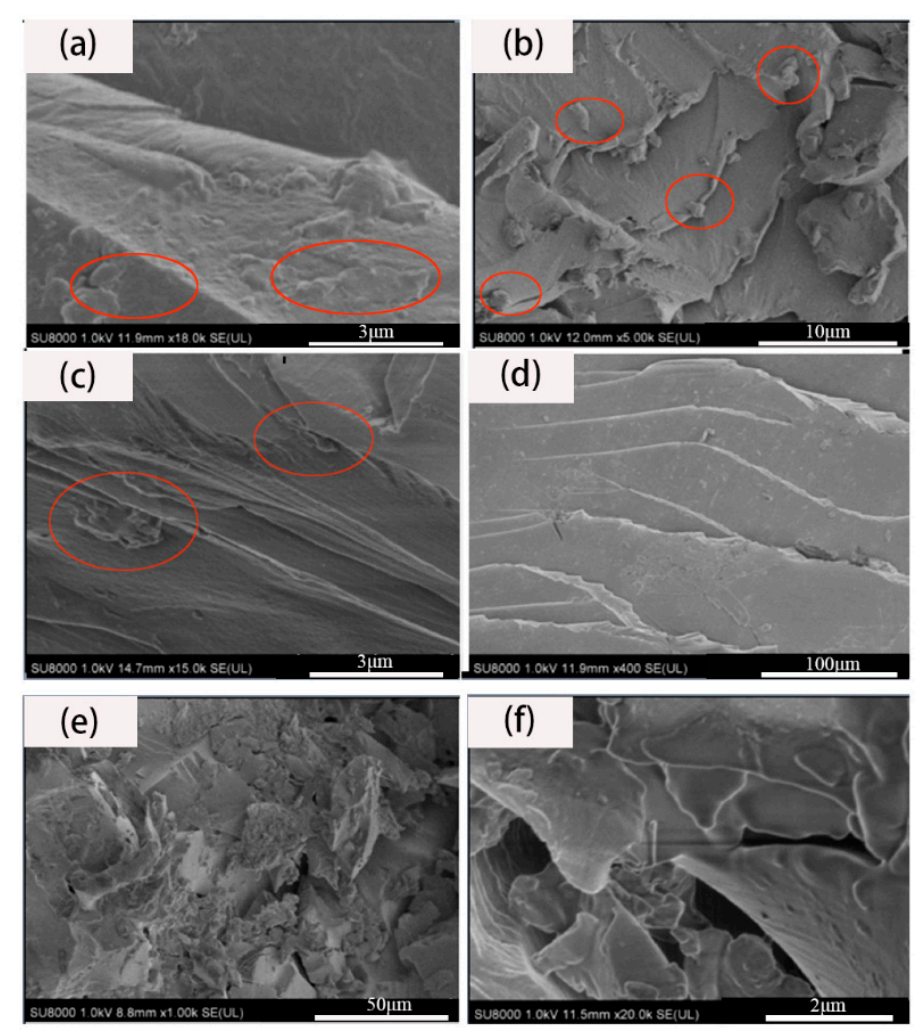

Figure 9. SEM images of the fracture surfaces of the composites: (a) modified GE/EP-0.03, (b) modified GE/EP-0.05, (c) modified GE/EP-0.07, (d) modified GE/EP-0.1, (e) CB/EP-0.4, (f) Ag/EP-20.

The conductivity of the as-prepared modified GE/EP, CB/EP, and Ag/EP composites is measured by using a four-probe conductivity meter, and the resistivity is shown in Table 1 . The results show that the resistivity of modified GE/EP-0.01 is huge according to the fact that the value is beyond the limitation of the instrument. However, the resistivity of modified GE/EP-0.1 decreases to $52 \Omega \cdot \mathrm{cm}$ due to the increase of the modified GE content. This result can be ascribed to the excellent conductivity of the modified GE and its homogeneous dispersion in the EP matrix. The modified GE sheets can make contact with each other and the intervals between them are very small, facilitating the formation of the continuous conductive path in the composites [45,46]. Thus, the as-prepared modified GE/EP composites in this paper exhibit excellent conductivity. The resistivity of the CB/EP composite decreases from 857 to $95 \Omega \cdot \mathrm{cm}$ when increasing the CB content from $0.2 \mathrm{wt} \%$ to $1.0 \mathrm{wt} \%$. The resistivity of the $\mathrm{Ag} / \mathrm{EP}$ composite decreases from 763 to $102 \Omega \cdot \mathrm{cm}$ when increasing the Ag particles 
content from $10 \mathrm{wt} \%$ to $50 \mathrm{wt} \%$. These results indicate that the modified GE as the filler for the EP matrix is more efficient to improve the conductivity compared with CB and Ag particles. The percolation theory has been used to explain the conductive behaviors of GE-based composites [23]. When the modified GE forms enough conductive paths to transmit electrons in the modified GE/EP composite, its conductivity is improved according to the percolation theory. In addition, the resistivity of the modified GE/EP, CB/EP, and Ag/EP composites with different contents of fillers is shown in Figure 10. The resistivity of the modified GE/EP composites decreases sharply in the range of 0.01 wt $\%-0.03$ wt $\%$. The resistivity of $\mathrm{CB} / \mathrm{EP}$ and $\mathrm{Ag} / \mathrm{EP}$ composites decreases sharply in the range of $0.2 \mathrm{wt} \%-0.4 \mathrm{wt} \% \mathrm{CB}$ and $10 \mathrm{wt} \%-20 \mathrm{wt} \%$ Ag particles, respectively. Thus, it is inferred that the percolation threshold of modified GE/EP, CB/EP, and Ag/EP composites is in the range of $0.01 \mathrm{wt} \%-0.03 \mathrm{wt} \%, 0.2 \mathrm{wt} \%-0.4 \mathrm{wt} \%$, and $10 \mathrm{wt} \%-20 \mathrm{wt} \%$ fillers, respectively.

Table 1. The resistivity of modified GE/EP, CB/EP, and Ag/EP composites with different contents of fillers.

\begin{tabular}{ccc}
\hline Filler & Samples & Resistivity $(\mathbf{\Omega} \cdot \mathbf{c m})$ \\
\hline \multirow{4}{*}{ Modified GE } & Modified GE/EP-0.01 & $1.84 \times 10^{5}$ \\
& Modified GE/EP-0.03 & 1013 \\
& Modified GE/EP-0.05 & 534 \\
& Modified GE/EP-0.07 & 162 \\
& Modified GE/EP-0.1 & 52 \\
\hline & $\mathrm{CB} / \mathrm{EP}-0.2$ & 857 \\
$\mathrm{CB}$ & $\mathrm{CB} / \mathrm{EP}-0.4$ & 633 \\
& $\mathrm{CB} / \mathrm{EP}-0.6$ & 518 \\
& $\mathrm{CB} / \mathrm{EP}-0.8$ & 313 \\
& $\mathrm{CB} / \mathrm{EP}-1$ & 95 \\
\hline & $\mathrm{Ag} / \mathrm{EP}-10$ & 763 \\
& $\mathrm{Ag} / \mathrm{EP}-20$ & 464 \\
$\mathrm{Ag} / \mathrm{EP}-30$ & 237 \\
& $\mathrm{Ag} / \mathrm{EP}-40$ & 125 \\
$\mathrm{Ag}$ particles-50 & 102 \\
\hline
\end{tabular}
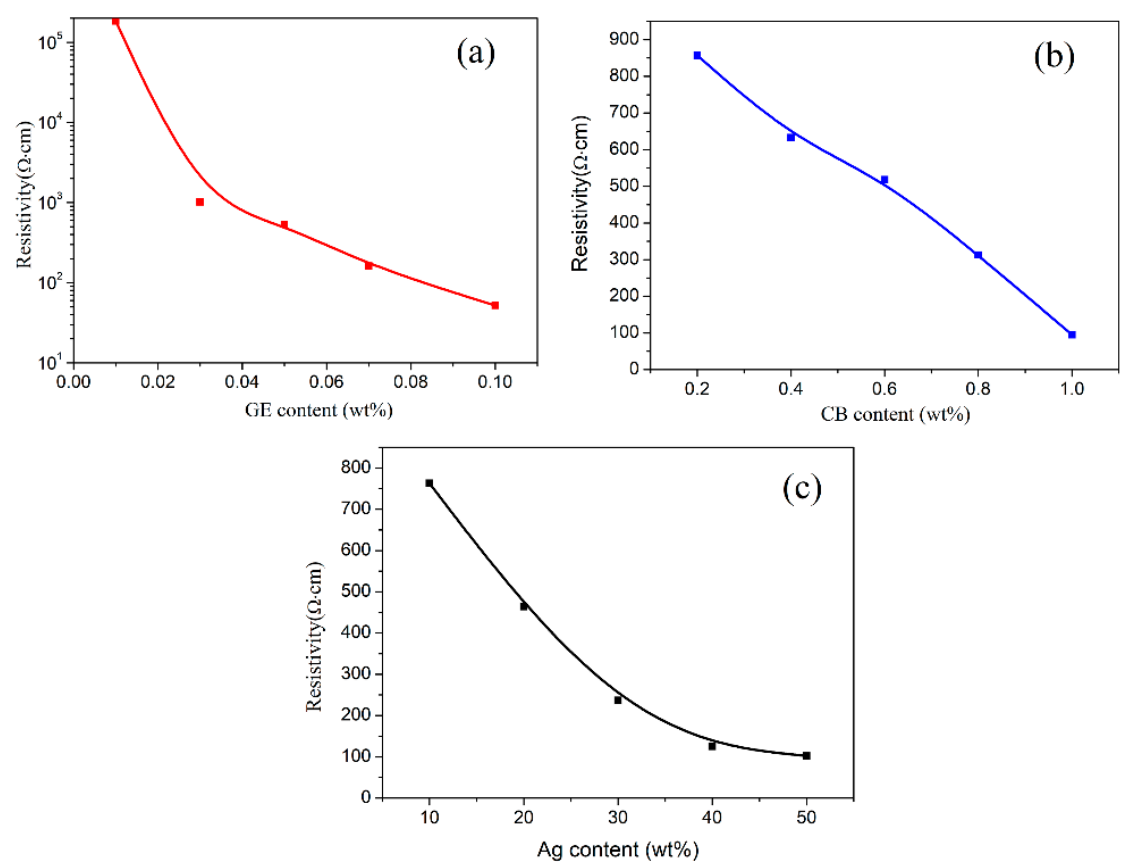

Figure 10. The resistivity of as-prepared modified GE/EP, CB/EP, and Ag/EP composites with different contents of fillers. 
For further estimating the percolation threshold of the modified GE/EP composite, the relation between the equated volume fraction of modified GE and the conductivity of as-prepared composites is showed in Table 2. Then, the experimental data is fitted according to the following power law model (Equation (4)):

$$
\sigma_{\mathrm{c}}=\sigma_{0}\left(\varphi-\varphi_{\mathrm{c}}\right)^{t}
$$

where $\sigma_{\mathrm{c}}$ is the conductivity of the composite, $\sigma_{0}$ is the conductivity of the conductive filler, $\varphi$ is the volume fraction of the filler, $\varphi_{\mathrm{c}}$ is the percolation threshold (the volume fraction), and $t$ is a universal critical exponent. As shown in Figure 11, the percolation threshold of the as-prepared modified GE/EP composites is $0.025 \mathrm{vol} \%$ modified GE by fitting the measured data (in Table 2) into Equation (4). When the volume fraction of the modified GE reaches the percolation threshold of $0.025 \mathrm{vol} \%$, the filler of the modified GE can form a conductive network to generate a leap increase in the conductivity of the composite. In addition, a linear relation is obtained for the log-log plot and it gives $t=1.8$ with a regression coefficient of 0.94 (as shown in the insert of Figure 11). The universal critical exponent of $t$ represents the dispersion dimension of the filler. The filler exhibits a random 3D dispersion when $t \geq 2$ and it exhibits a random 2D dispersion when $t<2[47,48]$. Moreover, the conductivity of the as-prepared modified GE/EP composites in this paper is comparable to that in previous reports, as shown in Table 3. It can be found that the modified GE content in our paper as the conductive filler is less and the as-prepared composites can show desirable conductivity.

Table 2. The conductivity of modified GE/EP composites with different volume fractions of modified GE according to the conversion of the mass.

\begin{tabular}{cc}
\hline Volume Fraction (vol \%) & Conductivity $\left(\mathbf{S} \cdot \mathbf{m}^{\mathbf{- 1}}\right)$ \\
\hline 0.012 & $5.4 \times 10^{-4}$ \\
0.037 & 0.099 \\
0.06 & 0.17 \\
0.085 & 0.62 \\
0.12 & 1.92 \\
\hline
\end{tabular}

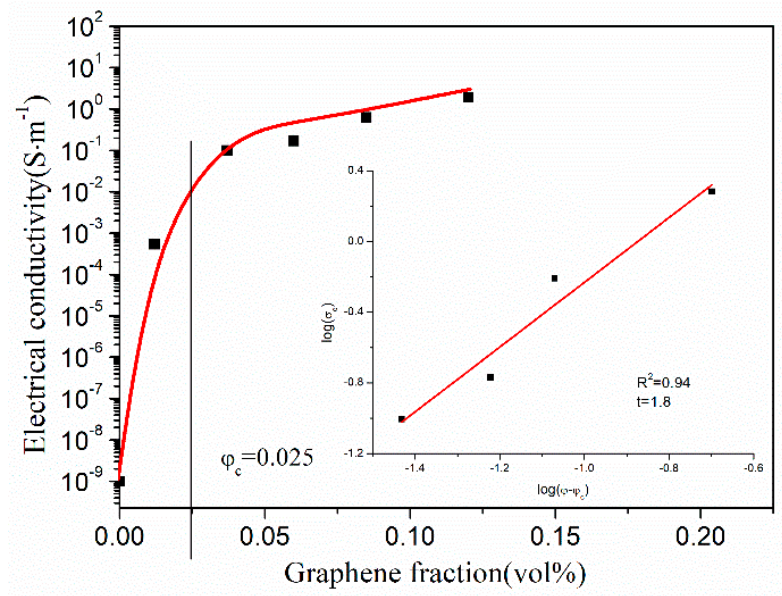

Figure 11. The conductivity of as-prepared modified GE/EP composites. Inset: log-log plot of illustrating the percolation model. 
Table 3. The conductivity of the polymer composites filled with different fillers in previous reports.

\begin{tabular}{cccc}
\hline Filler & Polymer Matrix & Content & Conductivity (S·m $^{\mathbf{- 1}}$ ) \\
\hline GE [10] & Polystyrene & $0.81 \mathrm{vol} \%$ & 15.7 \\
Epoxide-functionalized GE [49] & Epoxy & $0.48 \mathrm{vol} \%$ & $7.8 \times 10^{-8}$ \\
3D GE foam-PANI [50] & Epoxy & $0.39 \mathrm{wt} \%$ & 0.134 \\
GE-coated PU foam [51] & Epoxy & $0.9 \mathrm{wt} \%$ & 2.46 \\
Ag@CNTs [52] & Epoxy & $0.5 \mathrm{wt} \%$ & 0.8 \\
GE foam [53] & Epoxy & $0.2 \mathrm{wt} \%$ & 3 \\
GE [54] & Polyamide & $2.45 \mathrm{vol} \%$ & 60 \\
GE [55] & Epoxy & $13 \mathrm{wt} \%$ & 8.56 \\
\hline
\end{tabular}

\section{Conclusions}

The modified GE/EP composites with an excellent mechanical property and conductivity were prepared successfully using the double injection method in this paper. The optimized curing temperature of the modified GE/EP composites with a smooth surface is $75^{\circ} \mathrm{C}$. In addition, the total weight loss of modified GE/EP-0.05 is $26 \mathrm{wt} \%$ in the range of $50-600{ }^{\circ} \mathrm{C}$ and it exhibits a high thermal stability compared with pure EP. When the modified GE content increases to $0.07 \mathrm{wt} \%$, the tensile strength and bending strength of the composite can reach 74.65 and $106.21 \mathrm{MPa}$, respectively. When the modified GE content reaches the percolation threshold of $0.025 \mathrm{vol} \%$, it can form a conductive network in the EP matrix to generate a leap increase in the conductivity of the composite. The conductivity of the modified GE/EP composite reaches $1.92 \mathrm{~S} \cdot \mathrm{m}^{-1}$ when the modified GE content is $0.12 \mathrm{vol} \%$. In addition, the resistivity of CB/EP-1 and $\mathrm{Ag} / \mathrm{EP}-50$ is 95 and $102 \Omega \cdot \mathrm{cm}$, respectively. Compared with CB and Ag particles, the modified GE content in the composite is very low and it has a potential application in conductive ink as the conductive filler.

Author Contributions: Conceptualization, Y.C. and C.F.; Methodology, K.X.; Software, J.S.; Validation, Y.C., C.F. and Q.Z.; Formal Analysis, Q.Z.; Investigation, L.A.; Resources, J.C.; Data Curation, Q.Z.; Writing-Original Draft Preparation, Q.Z.; Writing-Review and Editing, Y.C.; Visualization, D.L.; Supervision, C.F.; Project Administration, Y.C.; Funding Acquisition, C.F.

Funding: This research was funded by the National Natural Science Foundation of China (No. 51772243), Technology Innovation Programme of Xi'an University of Technology (No. 2016CX030), China Postdoctoral Science Foundation Funded Project (No. 2016M592824), the Science and Technology Plan of Yulin City (No. 2016-16-7), and the Science and Technology Plan of Beilin District (No. GX1710).

Acknowledgments: The authors thank Northwest institute of chemical industry for the measurement. The authors thank Zhen Wang and Mannan Yang for their technical assistance.

Conflicts of Interest: The authors declare no conflict of interest.

\section{References}

1. Sumfleth, J.; Buschhorn, S.T.; Schulte, K. Comparison of rheological and electrical percolation phenomena in carbon black and carbon nanotube filled epoxy polymers. J. Mater. Sci. 2011, 46, 659-669. [CrossRef]

2. Mazov, I.; Burmistrov, I.; Il'inykh, I.; Stepashkin, A.; Kuznetsov, D.; Issi, J.P. Anisotropic thermal conductivity of polypropylene composites filled with carbon fibers and multiwall carbon nanotubes. Polym. Compos. 2015, 36, 1951-1957. [CrossRef]

3. Manta, A.; Gresil, M.; Soutis, C. Predictive model of graphene based polymer nanocomposites: Electrical performance. Appl. Compos. Mater. 2017, 24, 281-300. [CrossRef]

4. Pan, Y.; Sahoo, N.G.; Li, L. The application of graphene oxide in drug delivery. Expert Opin. Drug Deliv. 2012, 9, 1365-1376. [CrossRef] [PubMed]

5. Service, R.F. Materials science. Carbon sheets an atom thick give rise to graphene dreams. Science 2009, 324, 875-877. [CrossRef] [PubMed]

6. Balandin, A.A.; Ghosh, S.; Bao, W.; Calizo, I.; Teweldebrhan, D.; Miao, F.; Lau, C.N. Superior thermal conductivity of single-layer graphene. Nano Lett. 2008, 8, 902-907. [CrossRef] [PubMed] 
7. Heersche, H.B.; Jarillo-Herrero, P.; Oostinga, J.B.; Vandersypen, L.M.; Morpurgo, A.F. Bipolar supercurrent in graphene. Nature 2007, 446, 56-59. [CrossRef] [PubMed]

8. Pullicino, E.; Zou, W.; Gresil, M.; Soutis, C. The effect of shear mixing speed and time on the mechanical properties of GNP/epoxy composites. Appl. Compos. Mater. 2017, 24, 301-311. [CrossRef]

9. Zhao, Y.H.; Wu, Z.K.; Bai, S.L. Study on thermal properties of graphene foam/graphene sheets filled polymer composites. Compos. Part A Appl. Sci. Manuf. 2015, 72, 200-206. [CrossRef]

10. Zhao, P.; Luo, Y.; Yang, J.; He, D.; Kong, L.; Zheng, P.; Yang, Q. Electrically conductive graphene-filled polymer composites with well organized three-dimensional microstructure. Mater. Lett. 2014, 121, 74-77. [CrossRef]

11. Zhao, Y.H.; Zhang, Y.F.; Bai, S.L.; Yuan, X.W. Carbon fibre/graphene foam/polymer composites with enhanced mechanical and thermal properties. Compos. Part B Eng. 2016, 94, 102-108. [CrossRef]

12. Kim, H.; Miura, Y.; Macosko, C.W. Graphene/polyurethane nanocomposites for improved gas barrier and electrical conductivity. Chem. Mater. 2010, 22, 3441-3450. [CrossRef]

13. Bai, H.; Li, C.; Shi, G. Functional composite materials based on chemically converted graphene. Adv. Mater. 2011, 23, 1089-1115. [CrossRef] [PubMed]

14. Guo, Z.; Song, L.; Boay, C.G.; Li, Z.; Li, Y.; Wang, Z. A new multiscale numerical characterization of mechanical properties of graphene-reinforced polymer-matrix composites. Compos. Struct. 2018, 199, 1-9. [CrossRef]

15. Chen, Y.; Li, D.; Yang, W.; Xiao, C.; Wei, M. Effects of different amine-functionalized graphene on the mechanical, thermal, and tribological properties of polyimide nanocomposites synthesized by in situ polymerization. Polymer 2018, 140, 56-72. [CrossRef]

16. Alam, A.; Meng, Q.; Shi, G.; Arabi, S.; Ma, J.; Zhao, N.; Kuan, H.C. Electrically conductive, mechanically robust, pH-sensitive graphene/polymer composite hydrogels. Compos. Sci. Technol. 2016, 127, 119-126. [CrossRef]

17. Govorov, A.; Wentzel, D.; Miller, S.; Kanaan, A.; Sevostianov, I. Electrical conductivity of epoxy-graphene and epoxy-carbon nanofibers composites subjected to compressive loading. Int. J. Eng. Sci. 2018, 123, 174-180. [CrossRef]

18. Ji, X.; Xu, Y.; Zhang, W.; Cui, L.; Liu, J. Review of functionalization, structure and properties of graphene/ polymer composite fibers. Compos. Part A Appl. Sci. Manuf. 2016, 87, 29-45. [CrossRef]

19. Novoselov, K.S.; Geim, A.K.; Morozov, S.V.; Jiang, D.A.; Zhang, Y.; Dubonos, S.V.; Grigorieva, I.V.; Firsov, A.A. Electric field effect in atomically thin carbon films. Science 2004, 306, 666-669. [CrossRef] [PubMed]

20. Reina, A.; Jia, X.; Ho, J.; Nezich, D.; Son, H.; Bulovic, V.; Dresselhaus, M.S.; Kong, J. Large area, few-layer graphene films on arbitrary substrates by chemical vapor deposition. Nano Lett. 2008, 9, 30-35. [CrossRef] [PubMed]

21. Sutter, P.W.; Flege, J.I.; Sutter, E.A. Epitaxial graphene on ruthenium. Nat. Mater. 2008, 7, 406-411. [CrossRef] [PubMed]

22. Zang, C.G.; Zhu, X.D.; Jiao, Q.J. Enhanced mechanical and electrical properties of nylon-6 composite by using carbon fiber/graphene multiscale structure as additive. J. Appl. Polym. Sci. 2015, 132, 41968. [CrossRef]

23. Dai, W.; Yu, J.; Wang, Y.; Song, Y.; Bai, H.; Nishimura, K.; Liao, H.W.; Jiang, N. Enhanced thermal and mechanical properties of polyimide/graphene composites. Macromol. Res. 2014, 22, 983-989. [CrossRef]

24. Zhang, Z.; Zhang, W.; Li, D.; Sun, Y.; Wang, Z.; Hou, C.; Chen, L.; Cao, Y.; Liu, Y. Mechanical and anticorrosive properties of graphene/epoxy resin composites coating prepared by in-situ method. Int. J. Mol. Sci. 2015, 16, 2239-2251. [CrossRef] [PubMed]

25. Lotya, M.; King, P.J.; Khan, U.; De, S.; Coleman, J.N. High-concentration, surfactant-stabilized graphene dispersions. ACS Nano 2010, 4, 3155-3162. [CrossRef] [PubMed]

26. Wei, D.; Li, H.; Han, D.; Zhang, Q.; Niu, L.; Yang, H.; Bower, C.; Andrew, P.; Ryhänen, T. Properties of graphene inks stabilized by different functional groups. Nanotechnology 2011, 22, 245702. [CrossRef] [PubMed]

27. Lee, C.L.; Chen, C.H.; Chen, C.W. Graphene nanosheets as ink particles for inkjet printing on flexible board. Chem. Eng. J. 2013, 230, 296-302. [CrossRef]

28. Shulga, Y.M.; Bskakoy, S.A.; Baskakova, Y.V.; Lobach, A.S.; Kabachkov, E.N.; Volfkpvich, Y.M.; Sosenkin, V.E.; Shulga, N.Y.; Nefedkin, S.I.; Kumar, Y.; et al. Preparation of graphene oxide-humic acid composite-based ink for printing thin film electrodes for micro-supercapacitors. J. Alloys Compd. 2018, 730, 88-95. [CrossRef] 
29. Nemala, S.S.; Kartikay, P.; Agrawal, R.K.; Bhargava, P.; Mallick, S.; Bohm, S. Few layers graphene based conductive composite inks for Pt free stainless steel counter electrodes for DSSC. Sol. Energy 2018, 169, 67-74. [CrossRef]

30. Fang, H.; Zhou, T.; Chen, X.; Li, S.; Shen, G.; Liao, X. Controlled preparation and characterization of nano-sized hexagonal $\mathrm{Mg}(\mathrm{OH})_{2}$ flame retardant. Particuology 2014, 14, 51-56. [CrossRef]

31. Hummers, W.S., Jr.; Offeman, R.E. Preparation of graphitic oxide. J. Am. Chem. Soc. 1958, 80, 1339. [CrossRef]

32. Petersen, M.R.; Chen, A.; Roll, M.; Jung, S.J.; Yossef, M. Mechanical properties of fire-retardant glass fiber-reinforced polymer materials with alumina tri-hydrate filler. Compos. Part B Eng. 2015, 78, 109-121. [CrossRef]

33. Zhu, J.; Liu, A.; Wang, D. Study on the synergistic lithium storage performance of Sn/graphene nanocomposites via quantum chemical calculations and experiments. Appl. Surf. Sci. 2017, 416, 751-756. [CrossRef]

34. Mahesha, G.T.; Shenoy, S.B.; Kini, V.M.; Padmarajia, N.H. Effect of fiber treatments on mechanical properties of Grewia serrulata bast fiber reinforced polyester composites. Mater. Today Proc. 2018, 5, 138-144. [CrossRef]

35. Tessonnier, J.P.; Barteau, M.A. Dispersion of alkyl-chain-functionalized reduced graphene oxide sheets in nonpolar solvents. Langmuir 2012, 28, 6691-6697. [CrossRef] [PubMed]

36. Noh, Y.J.; Kim, S.Y. Synergistic improvement of thermal conductivity in polymer composites filled with pitch based carbon fiber and graphene nanoplatelets. Polym. Test. 2015, 45, 132-138. [CrossRef]

37. Mannaï, A.; Saidi, S.; Gmati, F.; Mohamed, A.B. Effects of hydrogen peroxide on the electrical conductivity of graphite/polyaniline composites. Mater. Sci. Semicond. Process. 2016, 41, 350-357. [CrossRef]

38. Markervich, E.; Salitra, G.; Levi, M.D.; Aurbach, D. Capacity fading of lithiated graphite electrodes studied by a combination of electroanalytical methods, Raman spectroscopy and SEM. J. Power Sources 2005, 146, 146-150. [CrossRef]

39. Chen, L.; Li, N.; Zhang, M.; Li, P.; Lin, Z. Effect of preparation methods on dispersion stability and electrochemical performance of graphene sheets. J. Solid State Chem. 2017, 249, 9-14. [CrossRef]

40. Stankovich, S.; Dikin, D.A.; Piner, R.D.; Kohlhaas, K.A.; Kleinhammes, A.; Jia, Y.; Wu, Y.; Nguyen, S.T.; Ruoff, R.S. Synthesis of graphene-based nanosheets via chemical reduction of exfoliated graphite oxide. Carbon 2007, 45, 1558-1565. [CrossRef]

41. Qin, H.; Zhang, S.; Zhao, C.; Feng, M.; Yang, M.; Shu, Z.; Yang, S. Thermal stability and flammability of polypropylene/montmorillonite composites. Polym. Degrad. Stab. 2004, 85, 807-813. [CrossRef]

42. Qiu, F.; Hao, Y.; Li, X.; Wang, B.; Wang, M. Functionalized graphene sheets filled isotactic polypropylene nanocomposites. Compos. Part B Eng. 2015, 71, 175-183. [CrossRef]

43. Tang, L.C.; Wan, Y.J.; Yan, D.; Pei, Y.B.; Zhao, L.; Li, Y.; Wu, L.B.; Jiang, J.X.; Lai, G.Q. The effect of graphene dispersion on the mechanical properties of graphene/epoxy composites. Carbon 2013, 60, 16-27. [CrossRef]

44. Chhetri, S.; Adak, N.C.; Samanta, P.; Murmu, N.C.; Kuila, T. Functionalized reduced graphene oxide/epoxy composites with enhanced mechanical properties and thermal stability. Polym. Test. 2017, 63, 1-11. [CrossRef]

45. Radzuan, N.A.M.; Zakaria, M.Y.; Sulong, A.B.; Sahari, J. The effect of milled carbon fibre filler on electrical conductivity in highly conductive polymer composites. Compos. Part B Eng. 2017, 110, 153-160. [CrossRef]

46. Xu, H.; Gong, L.X.; Wang, X.; Zhao, L.; Pei, Y.B.; Wang, G.; Liu, Y.J.; Wu, L.B.; Jiang, J.X.; Tang, L.C. Influence of processing conditions on dispersion, electrical and mechanical properties of graphene-filled-silicone rubber composites. Compos. Part A Sci. Manuf. 2016, 91, 53-64. [CrossRef]

47. Bauhofer, W.; Kovacs, J.Z. A review and analysis of electrical percolation in carbon nanotube polymer composites. Compos. Sci. Technol. 2009, 69, 1486-1498. [CrossRef]

48. Noël, A.; Faucheu, J.; Rieu, M.; Viricelle, J.P.; Bourgeat-Lami, E. Tunable architecture for flexible and highly conductive graphene-polymer composites. Compos. Sci. Technol. 2014, 95, 82-88. [CrossRef]

49. Zhao, S.; Chang, H.; Chen, S.; Cui, J.; Yan, Y. High-performance and multifunctional epoxy composites filled with epoxide-functionalized grapheme. Eur. Polym. J. 2016, 84, 300-312. [CrossRef]

50. Wang, K.; Wang, W.; Wang, H.; Liu, L.; Xu, Z.; Fu, H.; Zhao, L.; Zhang, X.; Chen, L.; Zhao, Y. 3D graphene foams/epoxy composites with double-sided binder polyaniline interlayers for maintaining excellent electrical conductivities and mechanical properties. Compos. Part A Appl. Sci. Manuf. 2018, 110, 246-257. [CrossRef]

51. Xu, X.Z.; Lin, G.M.; Liu, D.Y.; Sui, G.X.; Yang, R. Electrically conductive graphene-coated polyurethane foam and its epoxy composites. Compos. Commun. 2018, 7, 1-6. 
52. Ma, P.C.; Tang, B.Z.; Kim, J.K. Effect of CNT decoration with silver nanoparticles on electrical conductivity of CNT-polymer composites. Carbon 2008, 46, 1497-1505. [CrossRef]

53. Jia, J.; Sun, X.; Lin, X.; Shen, X.; Mai, Y.W.; Kim, J.K. Exceptional electrical conductivity and fracture resistance of 3D interconnected graphene foam/epoxy composites. ACS Nano 2014, 8, 5774-5783. [CrossRef] [PubMed]

54. Wang, P.; Chong, H.; Zhang, J.; Lu, H. Constructing 3D graphene networks in polymer composites for significantly improved electrical and mechanical properties. ACS Appl. Mater. Interfaces 2017, 9, $22006-22017$. [CrossRef] [PubMed]

55. Chiang, T.H.; Liu, C.Y.; Lin, Y.C. The effect of an anhydride curing agent, an accelerant, and non-ionic surfactants on the electrical resistivity of graphene/epoxy composites. J. Appl. Polym. Sci. 2015, 132, 41975. [CrossRef]

(C) 2018 by the authors. Licensee MDPI, Basel, Switzerland. This article is an open access article distributed under the terms and conditions of the Creative Commons Attribution (CC BY) license (http:// creativecommons.org/licenses/by/4.0/). 